\title{
Diversificación del ahorro familiar en el sistema financiero peruano
}

\section{RESUMEN}

El ahorro monetario resultante del esfuerzo de una familia debe conservarse e incrementarse con rendimientos que permitan contar con mayor disponibilidad de fondos en el futuro. Para que el dinero aumente de valor, su rendimiento, medido por la tasa de interés anual efectiva, debe ser superior a la inflación; además, el riesgo puede controlarse adoptando decisiones de ahorro según las alternativas que ofrecen las entidades financieras peruanas autorizadas. La información analizada de los años 2009 y 2010, confirma que las tasas de interés del mercado local dependen del monto del dinero, el plazo del depósito, y la situación y perspectivas de la entidad financiera.

Palabras clave: ahorro, rendimiento, riesgo, diversificación, inflación.

FAMILY SAVINGS DIVERSIFICATION IN THE financial System in Peru

\section{ABSTRACT}

The monetary savings resulting from a family effort to preserve and increase in yields as achieving greater availability of funds in the future. For money to grow in value, performance, measured by the effective annual interest rate must be higher than inflation; in addition, the risk can be controlled by saving decisions according to the options offered by the peruvian financial institutions authorized. The information analyzed for the years 2009 and 2010 , confirms that interest rates in the local market depending on the amount of money, the term of the deposit, and the status and prospects of the bank

Keywords: savings, performance, risk, diversification, inflation

\section{INTRODUCCIÓN}

Una familia como una unidad económica básica, requiere de fondos, denominados también recursos financieros, para satisfacer determinadas necesidades de sus integrantes, quienes a su vez tienen capacidad de generar fondos. El número de integrantes y la estructura de la familia varían con el tiempo, al igual que la capacidad de generar nuevos fondos, así como la naturaleza y el costo de las necesidades a satisfacer.

Una cultura financiera saludable consiste en ahorrar fondos cuando se tiene capacidad de generarlos, para utilizarlos en épocas en que el requerimiento de fondos para cubrir necesidades sea mayor a los nuevos ingresos que pudiese obtener la familia. El flujo de dinero, tanto de generación de fondos como de uso de fondos, se da en el tiempo, en donde el dinero adquiere diferente capacidad de compra por efecto de la inflación, expresada esta por el aumento porcentual generalizado en el precio de los bienes y servicios de la economía.

En el presente artículo, con información pública disponible durante el periodo enero - marzo de 2011, se analizan los principales factores a tener en cuenta, desde el punto de vista financiero, para conservar y aumentar el valor del dinero de una familia, que permita satisfacer en un horizonte máximo de un año calendario las necesidades de sus integrantes.

Como aplicativo se incluyen escenarios que pudiesen influir en los próximos doce meses, en los resultados de la gestión de un determinado monto de ahorro, con el objetivo de obtener un adecuado rendimiento, según el nivel de riesgo percibido en las alternativas de depósitos que ofrece el sistema financiero peruano.

\section{RENDIMIENTO DEL AHORRO}

El ahorro es el excedente de dinero que, para el caso del presente artículo, genera una familia en un determinado tiempo, que puede ser tres años, diez años, etc. Es la diferencia entre los ingresos de efectivo que percibe

1 Profesor Principal de la Facultad de Ingeniería Industrial de la UNMSM. E-mail: nestor_sj@hotmail.com 
y los gastos que realiza una familia. Los ingresos provienen de remuneraciones o de honorarios por servicios prestados, utilidades de negocios o de inversiones en general, bonificaciones, herencia, premios, etc. Los gastos, comúnmente denominados egresos de efectivo, corresponden a la atención de necesidades básicas, como alimentación, vestido, vivienda, salud y educación, así como también entretenimiento, viajes, compra de bienes en general, entre otros, según las expectativas de cada familia.

Se puede ver el ahorro también como el remanente de dinero que proviene de la diferencia entre ingresos y gastos, que puede resultar también de dejar de gastar o de posponer algunos gastos, para después satisfacerlas con los fondos a rentabilizarse durante un plazo futuro planeado. Por ejemplo, una familia puede optar por no almorzar los fines de semana fuera de casa, sacrificar el viaje de vacaciones de medio año, postergar la renovación de los artefactos y mobiliario del hogar, etc., porque se fija como meta recibir el próximo año nuevo en una isla o viajar de vacaciones en unión de los integrantes de la familia. Así visto, el ahorro es un sacrificio que se hace en el presente con una expectativa futura mejor, la cual al realizarse, en el ejemplo, contribuirá a cumplir un sueño o simplemente integrar aún más a la familia [1].

Debe planificarse y cuidarse el ahorro, para que el capital, que es el monto ahorrado inicialmente, y sus rendimientos, es decir los intereses generados por ese capital, se acumulen y aumenten, para lo cual dichos rendimientos deben ser superiores a la pérdida de la capacidad adquisitiva del dinero, pérdida que es producida por la inflación, y deben estar libres de cualquier evento que ponga en peligro su disponibilidad, vale decir el riesgo.

\section{a) Tasas de rendimiento corriente y real}

Las tasas de interés con las que remuneran las entidades financieras peruanas a los depósitos son tasas corrientes y efectivas, dependen de la oferta y demanda del dinero del mercado, y para que resulten atractivas para los ahorristas deberían contemplar la inflación esperada para los próximos meses. El ahorrista para percibir la ganancia de dicha remuneración, a la tasa corriente debe deducir la tasa de inflación, dando lugar a la tasa de interés real, que es el rendimiento por encima o sobre la inflación.

La tasa de interés real (r) es la diferencia entre la tasa corriente (i) y la inflación ( $\boldsymbol{\varnothing})$, teniendo en cuenta que la inflación afecta tanto al capital como a los intereses. Para encontrar la tasa real, consideremos una unidad monetaria de capital, cuyo va- lor futuro dentro de un año, con la tasa corriente, resulta $(\mathbf{1 + i})$; luego a dicho valor se quita la inflación -vía descuento o actualización del dinero-, dividiendo entre $(\mathbf{1 + \boldsymbol { \varnothing } )}$ y al resultado se resta la unidad; obteniéndose la siguiente relación [5]:

$$
r=\frac{(i-\varnothing)}{(1+\varnothing)}
$$

\section{Depósitos de "bajo rendimiento"}

Como ejemplo, asumamos que una persona natural deposita la suma de S/. 10000 en una entidad financiera peruana que remunera por dicho depósito la tasa de $5,00 \%$ anual, estimándose para el horizonte planeado de un año una inflación de $2,00 \%$. Para simplificar el análisis se asume que los impuestos que afecten a las transacciones financieras y los gastos colaterales aplicables son mínimos.

El rendimiento del depósito, de 5,00\% anual, que es una tasa de interés corriente, incluye como componente a la inflación, que se estima en 2,00\% anual, con lo que la tasa de rendimiento real será positiva e igual a 2,94\% anual.

Para una tasa de interés de 5,00\% anual, el capital de $S / .10000$, al final de un año se convierte en $S / .10500$, monto que resulta de la suma del capital por S/.10 000 e intereses por S/.500. En ese año, como los precios se elevan en 2,00\%, para estimar el valor de S/.10 500 del año 1 equivalente a valores del año 0 -fecha en que se efectúa el depósito-, a dicho monto se descuenta la tasa de inflación; para lo cual el valor del año 1 se actualiza con la inflación, dando lugar al monto de S/. 10294 a valores equivalentes del año 0 , de donde resulta una tasa real de 2,94\% anual. Estos cálculos se pueden observar en la Figura 1.

De esta manera, el que ahorra o deposita S/.10 000, en un año obtiene intereses de $S / .500$, importe que en términos de capacidad de compra de la fecha en que se realizó el ahorro, el año 0, equivale a S/. 294 , de modo que la diferencia, es decir S/.206, es la pérdida por efecto de la inflación promedio del periodo analizado.

Luego, si la inflación fuese igual a 5,00\% anual-que es la tasa de interés del depósito-, a valores reales, el depósito conserva su capacidad de compra y no gana intereses; si la inflación fuese mayor a 5,00\% anual, el depósito pierde su valor; y si la inflación fuese menor a 5,00\% anual, el depósito conserva su valor y genera ganancia real por intereses. 
Figura 1. Rendimientos corrientes y rendimientos reales (un año)

\begin{tabular}{|c|c|c|}
\hline \multicolumn{2}{|c|}{ En términos corrientes (o nominales) } & En términos reales (o constantes) \\
\hline 10000 & 10500 & 10294 \\
\hline 0 & Añ̃o & Año \\
\hline Capital inicial (año 0): & 10000 & Capital final (año 1): \\
\hline Capital final (año 1): & 10500 & (capital inicial + intereses) \\
\hline & (capital inicial + intereses) & Capital final (equivalente al año 0): $\quad 10294$ \\
\hline Tasa de interés (Varia & oorcentual del capital): & Tasa de interés (Variación porcentual del capital): \\
\hline Tasa corriente & $5,00 \%$ & Tasa real $(r)=$ \\
\hline
\end{tabular}

Fuente: Elaboración propia.

\section{Depósitos de "alto rendimiento"}

Ahora, asumamos que a la persona del ejemplo anterior, siempre en el mercado peruano, no le resulta atractivo el rendimiento ofrecido por la entidad financiera, por lo que podría optar en colocar sus fondos en una entidad "de reconocido prestigio" que le ofrece pagar una tasa de interés mayor a la que paga la entidad anterior, digamos $10,00 \%$ anual.

En este escenario, para un rendimiento corriente de $10,00 \%$ anual y una inflación de $2,00 \%$ anual, la tasa real a obtenerse será de 7,84\% anual, resultado que es sustancialmente mejor que el esperado según el ejemplo anterior. Pero aquí, el depósito podría tener mayor posibilidad de pérdida, no por la inflación, sino por la eventualidad que la entidad receptora del depósito fracase. El tema se complica cuando una entidad, al margen de la Ley, promueve el ahorro de las personas, tipo cooperativas, juntas, etc. Es importante acotar que las entidades del sistema financiero peruano se encuentran bajo el ámbito de supervisión de la Superintendencia de Banca, Seguros y AFP (SBS) y los ahorristas, personas naturales (PPNN), cuentan con un Fondo de Seguro de Depósito (FSD) que protege el capital ahorrado y los intereses generados hasta un determinado límite [2].

Entonces nos encontramos ante un tema de riesgo, que implica la posibilidad de percibir -por los depósitos- un menor rendimiento al esperado o deseado, o simplemente, en el caso extremo, de asumir la pérdida parcial o total del capital ahorrado conjuntamente con los intereses por buscar mayores rendimientos; usualmente a mayor tasa de interés de rendimiento cabe la posibilidad de mayor riesgo. La tasa de interés a considerar como base para tomar una decisión de depositar los ahorros en una entidad financiera, debe ser la tasa de interés real más una prima o cobertura por riesgo.

\section{b) Tasas de rendimiento en moneda nacional y en moneda extranjera}

La tasa de interés real que se indica en el literal anterior, toma como base la tasa corriente en moneda nacional (MN), vale decir en nuevos soles. Para los depósitos en moneda extranjera (ME), usualmente el dólar estadounidense, previamente se debe analizar el efecto en la tasa de interés corriente de la variación del tipo de cambio, que es el cambio en el precio del dólar estadounidense en nuevos soles, durante un determinado periodo, y luego estimar la tasa de interés real equivalente en nuevos soles.

Para analizar el efecto de la variación en el precio del dólar estadounidense, se puede tomar como referencia el valor promedio entre los tipos de cambio compra y venta. La variación porcentual entre el precio a una fecha futura y a una fecha presente, puede ser nula, positiva o negativa. Si el precio del dólar estadounidense aumenta, dicha variación es positiva, por lo que el nuevo sol se ha devaluado, lo que quiere decir que se requiere mayor cantidad de nuevos soles para comprar la misma cantidad de dólares estadounidenses, y en cambio el dólar estadounidense se aprecia, se ha fortalecido frente al nuevo sol. Pero si el precio del dólar estadounidense disminuye, por ser la variación del tipo de cambio negativa, el nuevo sol se aprecia y el dólar estadounidense se ha devaluado. Esta variación del tipo de cambio afecta tanto al capital como a los intereses del ahorro.

Para encontrar una relación numérica entre la tasa de interés corriente en dólares estadounidense [i(US\$)] y su equivalente en tasa de interés real en nuevos soles [r(Equiv.S/.)], se convierte previamente la tasa corriente en dólares estadounidense a su equivalente en nuevos soles a valores corrientes, y después dicha tasa se transforma en tasa de interés real; tal como se puede apreciar en la Figura 2. 
Figura 2. Conversión de tasas de interés de ME a su equivalente en MN

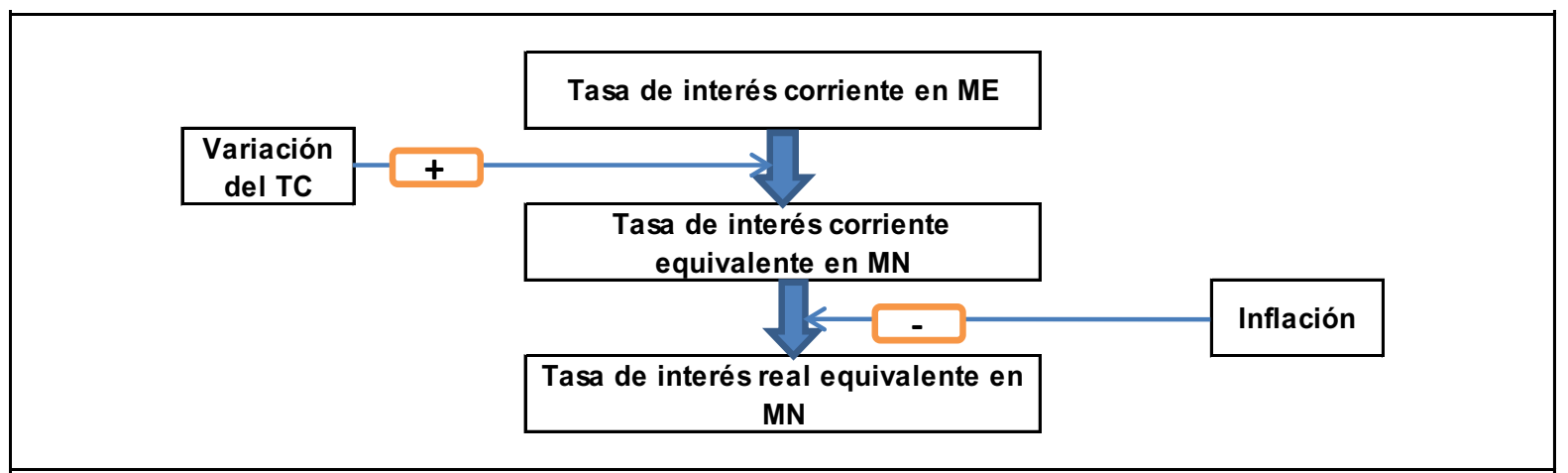

Fuente: Elaboración propia.

Para una variación porcentual del tipo de cambio (d), la tasa de interés corriente equivalente en nuevos soles será:

$$
\mathrm{i}(\text { Equiv.S/.) }=[(1+\mathrm{i}(\mathrm{US} \$)][1+\mathrm{d}]-1
$$

\section{Depósitos en dólares estadounidenses con "ga- nancia por tipo de cambio"}

Tomemos como ejemplo que una persona deposita una cantidad de dólares en una entidad financiera peruana que le remunera con una tasa de interés de $3,00 \%$ anual. El tipo de cambio actual de S/. 2,80/ US\$, dentro de un año de 360 días comerciales se eleva a S/. 2,94/US\$, con lo cual el dólar estadounidense se habría apreciado $5,00 \%$ con respecto al nuevo sol. Así la tasa de interés anual equivalente en nuevos soles será: $(1+0,03)^{\star}(1+0,05)-1=8,15 \%$ anual. El rendimiento en dólares estadounidenses de $3,00 \%$ anual, por efecto de la variación positiva del tipo de cambio, que es una ganancia, da lugar a un rendimiento de $8,15 \%$ anual en su equivalente en nuevos soles.

\section{Depósitos en dólares estadounidenses con "pérdida por tipo de cambio"}

Pero, si en ejemplo anterior, el tipo de cambio dentro de un año, luego de realizado el depósito de dólares estadounidense se reduce a S/. 2,74/US\$, que implica una disminución de $2,00 \%$ anual, se tendría una pérdida por diferencia de cambio. La tasa de interés en dólares estadounidenses de 3,00\% anual, dará lugar a que la tasa de interés equivalente en nuevos soles sea: $(1+0,03)^{*}(1-0,02)-1=0,94 \%$ anual.

Por lo tanto, para comparar el rendimiento de un depósito en $\mathrm{ME}$, en dólares estadounidenses, con el rendimiento de un depósito en $\mathrm{MN}$, es fundamental contar con el estimado de la variación del tipo de cambio durante el horizonte planeado para la decisión.

\section{RESULTADOS DE LOS ÚLTIMOS 12 MESES}

El conocimiento de la evolución histórica de los principales factores que influyen en el valor del dinero, medido este valor por la tasa de interés, que es la variación porcentual del dinero por cada unidad de tiempo -usualmente un año-, ayuda a analizar las alternativas de ahorro en el sistema financiero peruano a considerar para la obtención en el futuro de rendimientos adecuados para el capital disponible.

\section{a) Tasas de interés, inflación y tipo de cambio}

Durante el año 2010 las tasas de interés, en \% anual efectiva, de depósitos a plazo, del sistema financiero local en MN han sido superiores a las tasas de interés en ME, excepto en el mes de agosto, según se puede observar en la Figura 3 [3].

La tasa de interés promedio del año 2010 en MN, es $1,71 \%$ anual, la cual es menor a la inflación del indicado año, que fue de 2,10\% anual, expresada por la variación del Índice de Precios al Consumidor (IPC) de Lima Metropolitana, publicada por el Instituto Nacional de Estadística e Informática (INEI). De esta manera, en promedio, los rendimientos reales en el año 2010 han sido negativos, por lo que los depositantes en el sistema financiero peruano han perdido parte de su capital, al ser el rendimiento del depósito menor a la tasa de inflación.

La situación para el caso de depósitos en dólares estadounidenses durante el año 2010, es más que preocupante, donde el rendimiento promedio ha sido $0,87 \%$ anual, menor que la inflación, de 2,10\% anual, y antes de considerar el efecto de la variación del tipo de cambio. El tipo de cambio promedio compra venta al 31 de diciembre de 2009 fue de 
Figura 3. Tasas de interés promedio de depósitos a plazo (\% anual) - Año 2010

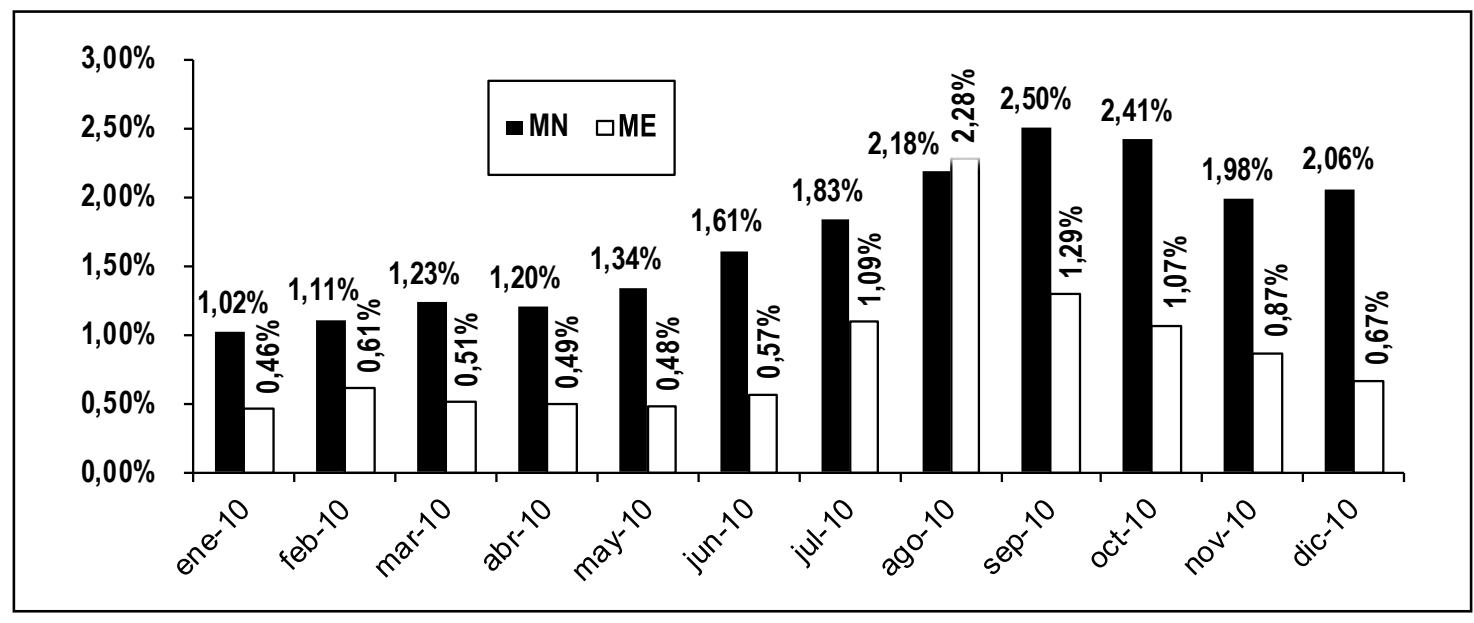

Fuente: INEI. Elaboración propia.

S/. 2,88950/US\$, el cual al cierre del año 2010 se redujo a S/. 2,80855/US\$, es decir el dólar de depreció en $2,80 \%$ anual.

De esta manera, en promedio, durante el año 2010, los depósitos a plazo en dólares estadounidenses tuvieron una doble pérdida en relación a depósitos en nuevos soles, una por la disminución del tipo de cambio, y otra por la inflación; en términos relativos, los depósitos a plazo en nuevos soles tuvieron menores pérdidas al considerarse la inflación.

\section{b) Alternativas de depósitos en el sistema finan- ciero peruano}

Las entidades financieras locales, conformadas por empresas bancarias, empresas financieras, cajas municipales de ahorro y crédito, y cajas rurales de ahorro y crédito, captan depósitos del público, bajo las modalidades de ahorro, depósitos a plazo y compensación por tiempo de servicios, en $\mathrm{MN}$ y en $\mathrm{ME}$, diferenciando a los depositantes en personas naturales y personas jurídicas. Entre los depósitos a plazo, se establecen periodos de hasta 30 días, 31-90 días, 91-180 días, 181-360 días y más de 360 días.

Las tasas de interés son fijadas libremente por las entidades financieras, por modalidad de operación, y son reportadas diariamente a la SBS, quien determina el promedio ponderado de las tasas de interés por modalidad de operación y por entidad financiera, y las publica diariamente en su página web y en los principales medios de comunicación. El promedio de la tasa de interés anual, para una base de 360 días, corresponde al promedio ponderado de las operaciones realizadas en el sistema financiero en los últimos 30 días útiles.
Para el análisis, bajo un criterio conservador, nos concentraremos en las oportunidades de ahorro o depósitos en general que ofrecen las empresas bancarias y empresas financieras. Las cajas municipales de ahorro y crédito y las cajas rurales de ahorro y crédito, si bien ofrecen rendimientos que superan a las tasas de interés de los bancos y las financieras, se encuentran en etapa de consolidación y eventualmente podrían implicar mayores riesgos, como para comprometer los ahorros de la familia, siempre que el monto del depósito por entidad no supere el importe cubierto por el FSD.

Como ilustración se tienen las tasas de interés para depósitos a plazo a más de 360 días, que es el plazo más cercano al año cronológico de 365 días. En la Tabla 1 se observan las tasas de interés vigentes al 30 de diciembre de los años 2009 y 2010, para depósitos en $\mathrm{MN}$ y en ME, y para personas naturales.

Se tiene que de un total de quince entidades bancarias, doce ofrecen depósitos a plazo, a una tasa de interés promedio que es menor a la de las empresas financieras, quienes de un total de nueve entidades, cuatro ofrecen este tipo de depósitos. Para ambas entidades, la tasa de interés en MN es superior a la tasa en ME.

A nivel de bancos y en MN, a diciembre de 2010, tres entidades: BBVA Banco Continental, Scotiabank Perú e Interbank, remuneran con una tasa promedio menor a $3,00 \%$ anual; por lo que, si la inflación que se registre en los próximos doce meses fuese superior a $3,00 \%$ anual, la tasa real resultará negativa, ocasionando la pérdida parcial del capital depositado. El Banco de Comercio ofrece la mayor tasa de interés, de $8,46 \%$ anual, seguido de 
Tabla 1. Tasas de interés, depósitos a plazo mayor a 360 días (PPNN)

en $\%$ anual

\begin{tabular}{|c|c|c|c|c|}
\hline \multirow{2}{*}{ Entidad Financiera } & \multicolumn{2}{|c|}{ Moneda Nacional } & \multicolumn{2}{|c|}{ Moneda Extranjera } \\
\hline & 30-dic-10 & 30-dic-09 & 30-dic-10 & 30-dic-09 \\
\hline \multicolumn{5}{|c|}{ Sistema bancario (Empresas bancarias) (Banca múltiple): } \\
\hline 1. BBVA Banco Continental & $2,69 \%$ & $1,35 \%$ & $1,32 \%$ & $1,64 \%$ \\
\hline 2. Comercio & $8,46 \%$ & $8,76 \%$ & $2,46 \%$ & $3,03 \%$ \\
\hline 3. Crédito & $3,85 \%$ & - & $1,65 \%$ & - \\
\hline 4. Financiero & $7,27 \%$ & $7,67 \%$ & $3,98 \%$ & $3,65 \%$ \\
\hline 5. Banco Interamericano & $4,22 \%$ & $2,71 \%$ & $3,25 \%$ & $3,37 \%$ \\
\hline 6. Scotiabank Perú & $1,09 \%$ & $1,19 \%$ & $0,98 \%$ & $0,93 \%$ \\
\hline 7. Citibank Perú & $5,18 \%$ & $5,20 \%$ & $0,53 \%$ & $1,23 \%$ \\
\hline 8. Interbank & $1,89 \%$ & $1,48 \%$ & $1,15 \%$ & $1,28 \%$ \\
\hline 9. MIBANCO & $6,60 \%$ & $7,57 \%$ & $3,16 \%$ & $3,94 \%$ \\
\hline 10. HSBC Bank Perú & $5,42 \%$ & $3,97 \%$ & $3,68 \%$ & $2,67 \%$ \\
\hline 11. Falabella Perú S.A. & $7,05 \%$ & $7,25 \%$ & $3,88 \%$ & $4,73 \%$ \\
\hline 12. Santander Perú & - & - & - & - \\
\hline 13. Banco Riplay Perú S.A. & $6,92 \%$ & $7,45 \%$ & $2,10 \%$ & $3,25 \%$ \\
\hline 14. Azteca del Perú & - & - & - & - \\
\hline 15. Deutsche Bank Peru & - & - & - & - \\
\hline Promedio Sistema Bancario & $5,13 \%$ & $4,77 \%$ & $1,42 \%$ & $1,31 \%$ \\
\hline \multicolumn{5}{|l|}{ Empresas financieras $\left(^{*}\right):$} \\
\hline 1. Crediscotia Financiera & $5,64 \%$ & $5,81 \%$ & $2,99 \%$ & $2,98 \%$ \\
\hline 2. Amérika Financiera & - & - & - & - \\
\hline 3. Financiera TFC S.A. & $4,10 \%$ & $2,80 \%$ & - & - \\
\hline 4. Financiera Universal & - & - & - & - \\
\hline 5. Financiera Uno S.A. & - & - & - & - \\
\hline 6. Financiera Edyficar & $3,62 \%$ & $1,55 \%$ & $1,05 \%$ & $1,05 \%$ \\
\hline 7. Financiera Crear & - & - & - & - \\
\hline 8. Financiera Confianza & $6,82 \%$ & - & $3,14 \%$ & - \\
\hline 9. Financiera Efectiva & - & - & - & - \\
\hline Promedio Empresas Financieras & $5,67 \%$ & $5,72 \%$ & $2,34 \%$ & $2,96 \%$ \\
\hline
\end{tabular}

${ }^{*}{ }^{*}$ Las tasas se aplican par depositantes personas Naturales y Personas Jurídicas.

Fuentes: SBS. Elaboración propia

Ios Bancos Financiero, MIBANCO y Falabella Perú S.A., con los cuales se espera un rendimiento real interesante, teniendo en consideración que la meta actual del Banco Central de Reserva (BCR) para la inflación, está entre $1,00 \%$ y 3,00\% anual.

\section{b) Protección de depósitos de personas naturales}

Para una persona natural, que no dispone de montos elevados como excedente de dinero, y no cuenta con especialización y/o tiempo suficiente para realizar análisis fundamentales y de situación y perspectivas del mercado del dinero, el sistema financiero local es una buena opción para rentabilizar el capital ahorrado, con un riesgo que se puede considerar razonable, dada la protección que ofre- ce a el FSD. Para montos mayores, por ejemplo al importe cubierto por el FSD, es deseable considerar operaciones en la Bolsa de Valores de Lima, Fondos Mutuos, AFP y/o depósitos en el exterior, con el apoyo de especialistas en administración de fondos.

El Estado peruano, a las personas naturales que depositan sus fondos en el sistema financiero local, los protege a través del FSD. Dicho Fondo se rige por las disposiciones contenidas en la Ley $\mathrm{N}^{\circ}$ 26702 "Ley General del Sistema Financiero, Sistema de Seguros y Orgánica de la SBS", y sus modificatorias, su Reglamento, su Estatuto y demás disposiciones que emita la SBS. Es una institución cuyo objetivo es proteger a los depositantes en 
caso la institución financiera miembro del FSD donde mantiene sus depósitos quiebre. Cuando la SBS interviene una institución financiera, el FSD paga un seguro a los depositantes equivalente al monto de su depósito más los intereses generados hasta por un monto que no puede superar el monto máximo de cobertura vigente.

EI FSD está integrado por las empresas bancarias, empresas financieras, cajas municipales de ahorro y crédito y cajas rurales de ahorro y crédito. Las Entidades de Desarrollo de la Pequeña y Micro Empresa (EDPYMES) serán miembros del FSD cuando se les autorice captar ahorros del público.

\section{PROPUESTA}

La data histórica, de por lo menos los últimos doce meses, es una referencia básica para decidir sobre el destino del monto ahorrado que se quiere rentabilizar en un periodo futuro máximo de un año; pero, lo determinante es el pronóstico con que se cuente para el horizonte planeado, en cuanto a inflación, tipo de cambio, tasas de interés, y en general las perspectivas del sistema financiero peruano.

Como proyecciones para la toma decisiones tenemos que la inflación para el año 2011, según la meta del BCR varía entre 1,00\% y 3,00\% anual, y el tipo de cambio promedio compra y venta, según algunos analistas financieros, al cierre del año 2011 podría ubicarse en el orden de S/. 2,75/US\$, lo que implica una reducción de $2,10 \%$ con respecto al valor registrado al 31 de diciembre de 2010 de S/. $2,81 /$ US $\$$. Entre otras fuentes de información, tenemos las proyecciones del Ministerio de Economía y Finanzas (MEF) y del BCR, contenidas en documento Marco Macroeconómico Multianual (MMM), el cual usualmente se aprueba en mayo y se revisa en agosto de cada año.

Los pronósticos varían según la fuente de información y naturalmente los resultados reales pueden diferir del valor pronosticado. Como ilustración en la Tabla 2 se presentan, según el MMM, los valores de la inflación y del tipo de cambio, real para el año 2009, estimado para 2010 y proyectado para los años 2011 al 2013 [4].
Si se revisan los resultados reales del año 2010, se tiene que la inflación, de 2,10\% anual, ha sido menor al estimado, que fue 2,70\%; al igual que el tipo de cambio, que resultó S/.2,81/US\$, menor al estimado de S/.2,83/US\$. Las proyecciones para los años 2011 al 2013 son actualizados por el MEF, en coordinación con el BCR, según el impacto de economía internacional en los principales indicadores de la economía nacional y los planes previstos para el desarrollo del mercado legal.

\section{a) Depósitos en dólares estadounidenses}

De contarse con saldos disponibles en dólares estadounidenses, se podría seleccionar depósitos a plazo en ME si se contara con alguna obligación y/o un plan de compromisos futuros en dicha moneda, para no correr el riesgo de afrontar en el futuro situaciones de mayor tipo de cambio al utilizado en el momento de la conversión inicial de dólares estadounidenses a nuevos soles.

Para el año 2011, con la información disponible sobre la variación del tipo de cambio y las tasas de interés ofrecidas por las entidades financieras, los depósitos a plazo en ME no resultarían atractivos.

\section{b) Depósitos concentrados en nuevos soles}

\section{Para personas conservadoras}

Como el seguro que ofrece el FSD asciende a S/.89 477 (importe vigente durante el periodo diciembre 2010 - febrero 2011), para una cobertura apropiada del capital e intereses a generarse en un año, y si el depositante -persona natural- tuviese fondos menores o iguales a S/. 85000 como referencia, lo recomendable es optar por depositar dicho monto en una sola entidad financiera. Para tal efecto, se sugiere:

1. Revisar las tasas de interés promedio publicadas en la página web de la SBS, en MN, a plazos mayores de 360 días, y seleccionar aquellas entidades entre el sistema bancario y las empresas financieras que remuneran con tasas de interés superiores a la máxima tasa de inflación esperada, de 3,00\%, más una cobertura por una tasa similar; con lo que se optaría por rendimientos mayores al 6,00\% anual.

Tabla 2. Inflación y tipo de cambio

\begin{tabular}{|c|r|r|r|r|r|}
\hline Concepto & $\mathbf{2 0 0 9}$ & $\mathbf{2 0 1 0}$ & $\mathbf{2 0 1 1}$ & $\mathbf{2 0 1 2}$ & $\mathbf{2 0 1 3}$ \\
\hline Inflación (\% anual) & $0,2 \%$ & $2,7 \%$ & $2,0 \%$ & $2,0 \%$ & $2,0 \%$ \\
Tipo de Cambio (S/./US\$) & 3,01 & 2,83 & 2,80 & 2,80 & 2,80 \\
\hline
\end{tabular}

Fuente: MEF y BCR. Marco Macroeconómico Multianual Revisado 2011-2013, aprobado agosto 2010. 
Según el Cuadro 1, las entidades a considerar serían: Banco de Comercio, con 8,46\%; Banco Financiero, con 7,27\%; MIBANCO, con 6,60\%; Banco Falabella Perú S.A., con 7,05\%; Banco Riplay Perú S.A., con 6,92\%; y Financiera Confianza, con $6,82 \%$ anual.

2. Visitar la página web de las entidades identificadas en el numeral anterior, para analizar las tasas aplicables para depósitos a plazo, teniendo en cuenta el monto disponible y el tiempo de permanencia del depósito en la entidad. Es posible encontrar tasas de interés atractivas, para dos o más entidades seleccionadas.

3. Negociar con el funcionario de las entidades seleccionadas, en forma directa, las mejores condiciones posibles y cerrar la operación con una de ellas. La capacidad de negociación del ahorrista depende del monto disponible y de sus antecedentes con la entidad financiera.

\section{Para personas que aceptan un mayor nivel de riesgo}

En este caso, la persona, su saldo disponible que supere el importe de S/. 85 000, podría colocarlo en una sola entidad financiera, la que remunere la mayor tasa de interés, con el riesgo de perder el importe del ahorro que supere al seguro que ofrece el FSD. Aquí se sugiere analizar la fortaleza de la entidad financiera, según los reportes que periódicamente publican las Empresas Clasificadoras de Riesgo de la entidad que sea de interés.

\section{c) Depósitos diversificados en nuevos soles}

Si el depositante contase con un saldo disponible por más de S/. 85 000, se pueden seguir los pasos mencionados anteriormente, y optar por colocar los fondos en dos o más entidades financieras, dependiendo del monto. Aquí se tendría lo siguiente:

\section{Para personas conservadoras}

Una persona que no esté dispuesta a asumir mayores riesgos, hasta el importe de S/. 85 000, podría depositarlo en la entidad que remunera la mayor tasa de interés y el saldo, por hasta un monto similar, en otra entidad financiera que remunere con una menor tasa, que sería la mayor de tasa de interés de los no seleccionados para el depósito de la primera parte.

\section{Para personas que disponen de mayores montos}

En general, si una persona cuenta con saldos disponibles superiores a S/. 85 000, lo saludable es que la persona diversifique sus depósitos en dos o más entidades financieras, cuya composición según entidades dependerá del nivel de riesgo que quiera asumir cada persona.

\section{CONCLUSIONES}

1. En el contexto macroeconómico actual y sus perspectivas para los próximos doce meses del periodo 2011-2012, el sistema financiero peruano ofrece interesantes oportunidades de rendimiento en nuevos soles para el capital excedente de las familias. Los depósitos a plazo en dólares estadounidenses no resultarían convenientes. Los depósitos a plazo en nuevos soles resultan atractivos, siempre que la remuneración que se pacte con la entidad financiera -bajo la supervisión de la SBS- se encuentre por encima de la inflación proyectada.

2. Para montos relativamente bajos, inferiores a la cobertura del FSD, las personas naturales pueden concentrar sus depósitos a plazo en una sola entidad financiera, aquella que ofrezca el mayor rendimiento promedio del sistema, mejor tarifario y según las condiciones que se pacten con el representante de la entidad. Para importes mayores a la cobertura del mencionado fondo, es conveniente diversificar los depósitos en dos o más entidades financieras del ámbito de la SBS, dependiendo del monto y la actitud del depositante ante el riesgo.

3. Una vez que la persona natural conservadora acumule depósitos a plazo en el sistema financiero peruano equivalente a cinco (05) importes del FSD en igual número de entidades financieras supervisadas por la SBS, sería recomendable la búsqueda de otras alternativas de ahorro y/o inversión, en donde se combinen expectativas de rendimiento y su aversión al riesgo.

\section{REFERENCIAS BIBLIOGRÁFICAS}

[1] Fernández, P. (2002). Valoración de empresas.

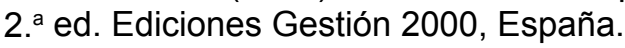

[2] FSD (2011). Depósitos en el sistema financiero nacional. http://www.fsd. org.pe/ (visitado el 2011-02-11).

[3] INEI (2011). Evolución de la inflación 10802011. http://www.inei. . .pe/ (visitado el 2011-0218).

[4] MEF (2011). Marco Macroeconómico Multianual 2011-2013 Revisado, aprobado en sesión de Consejo de Ministros del 25 de agosto de 2010. http://www.mef.gob.pe/contenidos/ 
pol_econ/marco_macro/MMM2011_2013_Rev. pdf (visitado 2011-02-18).

[5] Moyer, R. Ch., Mcguigan, J.R., Kretlow, W.J. (1998). Administración financiera contemporá- nea. Séptima edición, International Thomson Editores, México.

[6] SBS (2011). Tasas de interés activas en el sistema bancario. http://www.sbs.gob.pe/portalsbs/ tipotasa/ indice.htm (visitado el 2011-02-14). 